\title{
Two stage revision hip arthroplasty in periprosthetic joint infection. Comparison study: with or without the use of a spacer
}

\author{
Dariusz Marczak $^{1} \cdot$ Marek Synder $^{2} \cdot$ Marcin Sibiński $^{2} \cdot$ Michal Polguj $^{3} \cdot$ \\ Julian Dudka ${ }^{4}$ - Jacek Kowalczewski ${ }^{1}$
}

Received: 15 September 2016 / Accepted: 12 January 2017 / Published online: 18 May 2017

(C) The Author(s) 2017. This article is an open access publication

\begin{abstract}
Purpose The aim of this study was to compare two methods of two-stage surgery for PJI (periprosthetic joint infection) after THA (total hip arthroplasty): one with and one without the use of an antibiotic-loaded cement spacer.

Methods This retrospective study was performed on 99 consecutive patients (99 hips) with a minimum follow-up of 24 months. Patients were divided into two groups: (1) in
\end{abstract}

The protocol of the study was accepted by the Bioethics Committee of our institution (resolution RNN/43/13/KE dated 12 March 2013).

\section{Marcin Sibiński}

sibinskimar@gmail.com; sibinek@poczta.onet.pl;

marcin.sibinski@umed.lodz.pl

Dariusz Marczak

darmarczak@poczta.onet.pl

Marek Synder

msynder@pro.onet.pl

Michał Polguj

michal.polguj@umed.lodz.pl

Julian Dudka

zeromski@bci.pl

Jacek Kowalczewski

jackow@o2.pl

1 Orthopaedic Department, Postgraduate Medical Education Center, Otwock ul. Konarskiego 13, 05-400 Otwock, Poland

2 Department of Orthopaedics and Pediatric Orthopaedics, Medical University of Lodz, ul. Pomorska 251, 92-213 Łódź, Poland

3 Department of Angiology, Chair of Anatomy, Medical University of Łódź, ul. Narutowicza 60, 90-136 Łódź, Poland

$4 \quad$ Samodzielny Publiczny Zakład Opieki Zdrowotnej, Szpital Specjalistyczny im Stefana Żeromskiego, Kraków os. Na Skarpie 66, 31-913 Kraków, Poland whom the operation was performed using a spacer, and (2) for whom a spacer was not used.

Results For the whole cohort, the results improved between pre-operative and final follow-up. Recurrence of infection was found in nine out of 98 patients $(9.2 \%)$ and was not significantly different between the two groups. Patients treated with a spacer had better functional improvement in the interim period, but the VAS score was better in the non-spacer group. The improvement in final function was better in the spacer group with regard to HHS, but not according to WOMAC score or VAS at final follow-up.

Conclusion The resection arthroplasty should be awarded particular consideration in cases of poor soft tissue quality, bone stock deficiency, when complications related to spacer use are expected or chances of new hip endoprosthesis implantation are low.

Keywords Periprosthetic joint infection - Antibiotic therapy · Arthroplasty $\cdot$ Hip $\cdot$ Spacer

\section{Introduction}

Periprosthetic joint infection (PJI) after total hip arthroplasty (THA) presents a reconstructive challenge to the orthopaedic surgeon. The treatment goal is to attempt limb salvage, eradicate infection and preserve joint function. The prognosis for resolving infectious processes in hip arthroplasties ranges from 84 to $100 \%$ with current surgical techniques [1-3]. The two most common surgical procedures are one- and two-stage revisions. One-stage revisions, based on the immediate placement of a definitive prosthesis, are reserved for when the infecting organism has been identified and effective antibiotics are available. It can be the preferential method for infection with a single organism or with one of low virulence. Two- 
stage exchange arthroplasty should be used in patients with systemic manifestations of infection, a sinus tract or poor soft tissue coverage, when no organism has been identified or the infecting organisms are antibiotic resistant $[4,5]$.

The implantation of hip spacers in the first stage of THR for PJI is accepted worldwide as the main method of treatment. However, few studies have compared patients treated with spacers with those treated without them to demonstrate the real benefits of using a spacer in two-stage surgery [6, 7]. Furthermore, despite many of the advantages of spacers, they have been associated with complications related to their use including spacer dislocation, migration of the spacer to the pelvis following injury of the iliac vessels, spacer breakage and bone fracture on its removal $[6,8]$.

The aim of this study was to compare two methods of twostage surgery for PJI after THA: one with and one without the use of an antibiotic-loaded cement spacer.

\section{Materials and methods}

This was a retrospective study performed on 99 consecutive patients (99 hips) treated for PJI with two-stage revision THA. The first revision was performed from 2006 to 2014 in the place of work of the first author. The average follow-up period was 52.2 months (range, 26-120 months) from first stage revision to final follow-up, and at least 24 months from the second stage revision. Patients were divided into two groups on the basis of spacer use during the first stage of revision. For the first group (the study group with 47 patients), the operation was performed with the use of a spacer. The second group (control group with 52 patients) was operated on without any spacer. Both sets of patients had been treated at the same medical centre. The decision to use a spacer rested on the decision of the surgeon regarding the type of bone loss that can result in spacer-related complications. The procedures were carried out by five different surgeons.

The following inclusion criteria were required: (1) previous THA, (2) diagnosis of infection based on two positive periprosthetic cultures with phenotypically identical organisms, (3) sinus tract communicating with the joint, (4) informed consent obtained from patients for participation in the study.

Different exclusion criteria were used to assess reinfection rate and final results. For assessment of reinfection, only one patient from the control group was excluded due to not attending the follow-up. Other exclusion criteria were used for final result assessment. In the spacer group, two patients were excluded from the study as the spacer was left in and the secondstage operation was not performed. Neither patients agreed to the final surgery. Another four patients were excluded from the control group: one was lost to follow up and the other three were left with hanging hip; the patients refused further surgery. After the excluding process, 93 patients were included for the final analysis of results: 45 cases in the study group and 48 in the control group.

The demographic and clinical data of the patients are presented in Table 1.

The decision concerning revision surgery was made on the basis of clinical examination, presence of sinus tract communicating with the joint, radiographic imaging, positive periprosthetic or sinus tract cultures, and levels of ESR (erythrocyte sedimentation rate) and CRP (C-reactive protein). Cultures were kept for five days to allow the identification of micro-organisms. Sonication was not used in any of our patients.

The standard treatment for patients was the two-stage revision for PJI after THA. A posterior approach was taken for all procedures. During the first stage, between three and six tissue and fluid samples were taken for analysis after approaching the hip. Any sinus was excised, and this was followed by wide debridement of infected material and excision of all necrotic, contaminated or friable tissue. The old implants were removed. Debridement of infected tissues, including bone cement if necessary, and washout were then performed with copious quantities of normal saline and Betadine. Finally, in the first group, a Vancogenx Spacer Hip (Tecres, Italy) was implanted without the use of bone cement; while in the second group, a gap was left without the spacer in the bony acetabulum and medullary canal of the femur after removal of implants and debridement. Commercially available spacers used in the study are loaded with gentamicin that is released to surrounding tissue. The decision to use a spacer was based on the preferences of the surgeon and local bone stock deficiency. For example, if the risk of dislocation was high due to lack of a posterior acetabulum, no spacer was used. The suction drain was left until the second day after the operation, or as long as purulent discharge was present.

The second stage was performed only when erythrocyte sedimentation rate, white blood cell count and CRP were at normal levels. After a minimum period of two to three months, if no clinical signs of infection were observed, CRP level was below $10 \mathrm{mg} / \mathrm{dL}$, and white blood cell count and erythrocyte sedimentation rate were within normal ranges ( $<30 \mathrm{~mm} / \mathrm{h}$ ), second-stage revision was performed. During the second-stage operation, the spacer was removed (if present), and pulse-lavage performed by using water and washout and Betadine. In some patients, the spacer remained for a longer time than was primarily planned, for reasons independent of the surgeon.

Post-operative care was the same in both groups. The drains were removed on the second day after the operation. Intravenous antibiotics were applied for one week, and then oral antibiotics were prescribed for six weeks according to an antibiogram, or empirical information if the cultures were negative. After eight weeks of clinical examination, CRP and 
Table 1 Demographic and clinical data of patients treated with total hip arthroplasty for prosthetic joint infections with and without spacer

\begin{tabular}{|c|c|c|c|}
\hline Characteristic & $\begin{array}{l}\text { Group treated with spacer mean } \\
\text { (range) }\end{array}$ & $\begin{array}{l}\text { Group treated without spacer mean } \\
\text { (range) }\end{array}$ & $P$-value \\
\hline Number of patients & 45 & 48 & \\
\hline Male / female & $26 / 19$ & $30 / 18$ & 0.64 \\
\hline Age & 62.0 years $(24-83)$ & 64.6 years $(29-87)$ & 0.3 \\
\hline Time from primary to first-stage THA & 55.2 months $(5-264)$ & 32. months $8(3-108)$ & 0.013 \\
\hline Time from the first- to second-stage revision THA & 5.5 months $(2-16)$ & 14.5 months $(3-39)$ & $>0.00001$ \\
\hline Duration of first stage surgery & $138.8 \min (72-220)$ & $126.7 \min (56-207)$ & 0.17 \\
\hline Duration of second stage surgery & $146.1 \min (111-261)$ & $181.5 \min (66-295)$ & 0.0005 \\
\hline Length of hospital stay - first stage surgery & 12.3 days $(8-18)$ & 12.6 days $(8-17)$ & 0.4 \\
\hline Length of hospital stay - second stage surgery & 10.0 days $(6-14)$ & 10.4 days $(5-15)$ & 0.3 \\
\hline Type of bacteria & $\begin{array}{l}\text { MSSA - } 13 \\
\text { MRSA - } 11 \\
\text { Staphylococcus epidermidis }-6 \\
\text { Enterobacter cloacae }-1 \\
\text { Streptococcus }-2 \\
\text { Not found }-12\end{array}$ & $\begin{array}{l}\text { MSSA }-21 \\
\text { MRSA }-1 \\
\text { Staphylococcus epidermidis }-5 \\
\text { Enterococcus faecalis }-3 \\
\text { Streptococcus }-5 \\
\text { Klebsiella pneumoniae }-1 \\
\text { Not found }-12\end{array}$ & \\
\hline Bacteria detection rate & $46 \%$ & $63 \%$ & \\
\hline HHS before first revision & 56.1 points $(21.7$ to 74.3$)$ & 61.1 points $(45.6-71.4)$ & 0.12 \\
\hline WOMAC before first revision & 53.8 points (39.1-75.4) & 61.2 points (43.2-78.6) & 0.02 \\
\hline VAS before first revision & 7.0 points $(4-9)$ & 6.9 points $(3-9)$ & 0.74 \\
\hline HHS spacer/hanging hip & 66.1 points (43.5-76.8) & 58.5 points (48.9-68.8) & $>0.0001$ \\
\hline WOMAC spacer/hanging hip & 59.7 points (40.6-78.4) & 57.2 points (49.8-67.9) & 0.06 \\
\hline VAS spacer/hanging hip & 5.2 points $(2-8)$ & 4.0 points $(1-6)$ & 0.0006 \\
\hline HHS $1 / 2$ difference & 10.1 points $((-10.9)-36.2)$ & $(-2.6)$ points $((-17.5)-15.4)$ & $>0.0001$ \\
\hline WOMAC $1 / 2$ difference & 5.8 points $((-4.7)-21)$ & $(-4.0)$ points $((-27.1)-15.2)$ & 0.000001 \\
\hline Final HHS & 80.2 points (40.3-93.1) & 77.7 points $(61.5-89.7)$ & 0.06 \\
\hline Final WOMAC & 74.5 points (43.8-88.8) & 79.9 points (54.7-93.3) & 0.006 \\
\hline Final VAS & 2.7 points & 2.5 points & 0.5 \\
\hline HHS difference & 25.2 points $(1.9-49.8)$ & 16.6 points $((-2.6)-40.8)$ & 0.0005 \\
\hline WOMAC diff & 20.7 points $((-3.7)-39.3)$ & 18.6 points $((-1,3)-37)$ & 0.33 \\
\hline $\begin{array}{l}\text { Second stage (number of packs of concentrated red blood } \\
\text { cells) }\end{array}$ & $2.5(0-5)$ & $3.6(0-5)$ & 0.19 \\
\hline Reinfection & 6 (incl. 47 patients) & 3 (incl. 51 patients) & 0.23 \\
\hline
\end{tabular}

THA total hip arthroplasty, MSSA methicillin-sensitive Staphylococcus aureus, MRSA methicillin-resistant Staphylococcus aureus, HHS Harris hip score, WOMAC Western Ontario and McMaster Universities Arthritis Index, VAS visual analog scale

HHS, WOMAC $1 / 2$ difference indicates the difference in results before first revision and with spacer/hanging hip; HHS, WOMAC difference difference in results before first revision and at final follow-up

Final clinical and functional KKS score are calculated after excluding patients with recurrence of infection. Values in bold text indicate statistical significance

ESR examinations were performed, with the CRP examination repeated until normalization. All patients were evaluated radiographically at six-month intervals with standard anteroposterior hip radiographs in a standing position. In nine patients, normal CRP levels were not achieved and recurrence of infection was observed (six in the spacer group and three in the non-spacer group).

The Harris hip score (HHS) [9] and Western Ontario and McMaster Universities Arthritis Index (WOMAC) [10] were used for clinical assessment. Clinical and functional results were calculated only for patients without recurrence of infection.

\section{Statistical analysis}

Statistica for Windows $10 \mathrm{Pl}$ was used for statistical analysis. The chi-squared test was applied to calculate the significance of the difference between genders in both groups. Fisher's exact test was applied to compare infection rates in the two groups. Levene's test was used to test the normality of 
distribution. The U Mann-Whitney test was used to make the following comparisons between groups: HSS (initial and in the interim period), HSS score difference between final and initial results as well as between interim and initial results, WOMAC score in the interim period, WOMAC score difference between interim and initial results and time period form the first-stage to second-stage revision THA. The other comparisons between groups were performed using Student's Ttest for independent samples. Student's T-test for dependent samples was used to compare VAS, HHS and WOMAC for the whole cohort in different time periods.

\section{Results}

The members of the groups were comparable in terms of age and gender (Table 1). None of the patients were on immunosuppressive therapy, corticosteroid therapy or had diabetes. Recurrence of infection was found in nine out of 98 patients $(9.2 \%)$. Reinfection was detected in six patients from the spacer group and three (6.4\%) from the non-spacer group. The difference was not statistically significant $(p=0.23)$.

No statistically significant difference between time points was observed for mean WOMAC score for all 93 patients treated - a pre-operative score of 57.7 points compared to 58.4 following first revision $(p=0.4)$. Similarly, the preoperative score (57.7 points) was lower than the final follow-up score (77.3 points) $(p<0.001)$, as was the score following first revision (58.4) compared to final follow-up (77.3 points) $(p<0.001)$. There was also no difference between groups in terms of number of packs of concentrated red blood cells $(p=0.19)$.

A significant difference was found between pre-operative HHS score and HHS score after first revision for all patients (mean 58.6 versus 62.2 points, respectively; $p=0.002$ ). Preoperative HHS score was lower than at final follow up (mean 58.6 versus 79.4 points, respectively; $p<0.00001$ ), as was the score after first revision compared to final follow-up (mean 62.2 versus 79.4 points, respectively; $p<0.001$ ).

The mean VAS score improved from 6.9 pre-operatively to 5.1 points in the interim period $(p<0.001)$. The pre-operative score was lower than the final follow-up score ( 6.9 versus 2.6 points, respectively; $p<0.00001$ ), as was the score from the interim period and the final follow-up (5.1 versus 2.6 points, respectively; $p<0.001$ ).

One patient had a complication related to the spacer that was found to be subluxated on post-operative radiographs. The subluxated spacer was left for final second-stage revision. Five patients from the study group underwent spacer exchange together with debridement due to recurrence of infection. The infection was successfully eradicated in all those patients, and they were followed up with THA.
The comparison between groups given in Table 1 found that patients treated with spacer demonstrated better functional improvement in the interim period than those treated without spacer, with regard to mean HHS (10.1 versus $(-2.6)$ points, respectively; $p<0.00001)$ and mean WOMAC score $(5.8$ versus $(-4.0)$ points, respectively; $p<0.00001)$. Despite the fact that the final mean WOMAC score was better in the control group than the study group (mean 79.9 versus 74.5 points, respectively; $p<0.006$ ), greater improvement in functional status from pre-operative to final score was found in the spacer group according to HHS than in the non-spacer group (mean 20.2 versus 16.6 points, respectively; $p=0.0001$ ), but not according to WOMAC score (mean 20.7 versus 18.6 points, respectively; $p=0.33$ ). The VAS score in the interim period was better in the control group than in the study group (mean 4.0 versus 5.2 points, respectively; $p=0.03$ ), but not at final follow-up (mean 2.5 versus 2.7 , respectively; $p=0.6$ ) (Table 1).

\section{Discussion}

Several factors could be considered when examining the influence of the use of spacers in treating PJI after THR: reinfection rate, the interim and final results, duration of operation and length of hospital stay and complications related to spacer. However, any comparison of our results with others is complicated by the variation in the operative and peri-operative protocols and the small number of potential comparative studies $[6,7,11]$.

While some functional improvement was found in all patients even after the first revision, during which the implants were removed according to HHS, the most significant functional improvement was noted after second stage revision. In addition, the entire cohort reported significant functional improvement at the final visit compared to pre-operative status. The final results of the two stage procedure are satisfactory in terms of function, pain and eradication of infection $(9.2 \%)$. Two-stage reimplantations have previously been found to successfully eradicate infection in 84-100\% [1-3], which is consistent with our observations. A systematic review and metaanalysis based on aggregate published data found the reinfection rate after one- or two-stage revision to be approximately $8 \%$ [2]. Staphylococcus species were identified in about half of patients, which is consistent with findings provided by Kliushin et al. [12].

Several mechanical complications may occur when cement spacers are used: spacer fractures, dislocations (up to 16.4\%) and femoral fractures have frequently been reported [3, 8]. The present study found a much lower complication rate related to spacer use, with only one spacer dislocation identified. This may be related to the fact that a spacer was not used if dislocation was likely to occur. 
The reinfection rate was similar in both groups: $13.3 \%$ in the spacer group and $6.4 \%$ in the non-spacer group. One possible reason for this is that the spacer is a foreign body and thus may encourage colonization by bacteria. In some studies, biofilm has been found on the spacer removed from the hip [13-15].

Jahoda et al. also examined the use of a spacer in similar operations. The success rate in terms of reinfection was found to be similar in patients treated with a spacer (96.5\%) and those who were not $(94.3 \%)$ [7]. Hsieh et al. compared two types of hip PJI treatment which used either antibiotic-loaded cement beads or antibiotic-loaded cement prostheses in the first stage of the operation. Recurrence of infection was also similar in both groups [11]. Pagnano et al. found that resection arthroplasty was reliable in eradicating reinfection, but led to poor function and was associated with persistent pain [16]. A similar conclusion was reached by Castellanos et al., who found that infection was satisfactorily controlled in $86 \%$ of patients with Girdlestone pseudarthrosis after infected THR [17]. This finding contrasts with those reported in other studies. Cabrita et al. report a lower infection rate after first and second stage of revision with the use of a spacer compared to hanging hip [6].

The literature review of the effect of spacer use on final clinical results remains unclear. Cabrita et al. report no significant difference in functional results in patients treated with a two-stage procedure, either with a spacer or without [6]. In a study of hip function following Girdlestone pseudarthrosis, Schröder et al. conclude that the observed improvement in hip function following reimplantation was marginal and the results were comparable to a well-functioning pseudarthrosis [18]. It is worth noting that Schröder et al. used a significantly longer time period between revision stages (mean three years) than that employed in the present study. This results in more severe muscle contracture and changes in walking biomechanics, and hence the less favourable outcomes reported by Schröder et al. [18]. Blomfeldt et al. report high rate of Girdlestone operation performed in patients with PJI treated primary or secondary with arthroplasties for displaced femoral neck fracture and the outcome treatment was unfavourable [19].

In a comparison of antibiotic-loaded cement beads and antibiotic-loaded cement prosthesis by Hsieh et al., the use of a spacer was associated with a higher hip score, a shorter hospital stay, and better walking capacity in the interim period; a decreased operative time, less blood loss, and a lower transfusion requirement at the time of reimplantation; and fewer post-operative dislocations [11]. A similar method of treatment was employed by Takigami et al., who used porous hydroxyapatite blocks loaded with an antibiotic instead of spacers; they found that function improved from 45.1 before surgery to 79.6 at the latest follow-up according to the Japanese Orthopaedic Association [20]. Jahoda et al. report greater improvement in final results in a group of patients treated using a spacer (an improvement of 29 points) compared to those who were not (20 points). They also highlight that use of a spacer in two-stage reimplantation ensured greater comfort for the patient [7].

Both the initial and final clinical results in our study groups were significantly different, which made any comparison difficult and confusing. For this reason, we drew our conclusion on the basis of differences in outcomes between time periods. A more significant improvement in hip function was found in the group treated with spacer when initial and final results were compared according to HHS, but not WOMAC score. Those patients also demonstrated greater improvement in the interim period, i.e. the difference between the interim and initial outcomes, due to better range of motion, better walking ability and less leg length discrepancy; however, this cohort also reported more pain related to the spacer than the nonspacer group. Conversely, patients with resection arthroplasty complained of leg length discrepancy and hip instability, but reported less pain, which could explain the discrepancies between HHS and WOMAC scores at the analyzed stages. We believe that the greater improvement of HHS between the initial and final results observed in the study group was not related to the use of a spacer, but the choice of better bone stock and a shorter interim period. A significantly longer period between first and second stage revision was chosen for the patients treated without a spacer, and they reported less pain in the interim period and anxiety from recurrence of infection. They accepted some inconvenience related to walking difficulties and impaired function. Furthermore, to prevent spacer dislocation, the spacer was not implanted if severe bone deficiency was noted, especially in the posterior acetabulum.

Our findings confirm those of other authors, who report a longer duration for the second-stage operation if the spacer was not implanted during first stage revision $[6,7,11]$. Similarly, our findings indicate that reimplantation of hip endoprosthesis was about one hour longer if the spacer was not present; more time was needed to remove scar tissues both from the acetabulum and medullary canal. The surgeons tended to perform second stage realoplasty if a spacer had previously been used. In a prospective study comparing two groups of patients treated with and without spacer, Cabrita et al. found that when the spacer was used, the operation was around one hour shorter, it was easier to find surgical planes, identify the bone structures and build the bed for the implant, compared to the control group [6]. Similar findings are presented by Hsieh et al. [11]. We fully agree that it is difficult to dissect the muscular planes and identify bone landmarks. Finding the bony acetabulum, intramedullary femoral canal and removal of scar tissue is more challenging in those cases and takes more time. It is also worth noting that spacers were not used in our cohort in cases of more severe bone deficiency, especially the loss of bony acetabulum. Consequently, second stage revision requires more complex reconstruction methods, such as the use of cages, metal blocks or screws. 
The two groups were not found to be significantly different with regard to length of hospital stay during first and second stage revision. In some orthopaedic departments, it is a routine practice to use skeletal traction after the removal of implants from an infected hip, which significantly prolongs hospital stay [6]. A longer hospital stay associated with the use of spacer was also noted by Hsieh et al. [11].

This study has some limitations. First, this is a retrospective study and the data comes from medical records of patients. Second, this is a consecutive series of patients, but operated by five different surgeons. This doctors came from the same medical centre and had the same training program. Furthermore, cultures were kept for five days to allow the identification of micro-organisms, and sonication was not used in any of our patients. Nowadays we use sonication and keep the bacteria cultures much longer, which increases the bacteria type detection rate.

In summary, we believe that resection arthroplasty may be a valuable first-stage procedure in the treatment of PJI after THR. The success rate in terms of infection control is similar to that observed in operations with the use of a spacer. It should be especially considered in cases of poor soft tissue quality, bone stock deficiency, and when complications related to spacer use are expected or when the chances of new hip endoprosthesis implantation are low.

Compliance with ethical standards This research did not receive any specific grant from funding agencies in the public, commercial, or notfor-profit sectors.

All procedures performed in studies involving human participants were in accordance with the ethical standards of the institutional and/or national research committee and with the 1964 Helsinki declaration and its later amendments or comparable ethical standards.

Informed consent was obtained from all individual participants included in the study.

\section{Conflict of interest All authors declare no conflict of interest.}

Open Access This article is distributed under the terms of the Creative Commons Attribution 4.0 International License (http:// creativecommons.org/licenses/by/4.0/), which permits unrestricted use, distribution, and reproduction in any medium, provided you give appropriate credit to the original author(s) and the source, provide a link to the Creative Commons license, and indicate if changes were made.

\section{References}

1. Klouche S, Leonard P, Zeller V, Lhotellier L, Graff W, Leclerc P et al (2012) Infected total hip arthroplasty revision: one- or twostage procedure? Orthop Traumatol Surg Res 98:144-150

2. Kunutsor SK, Whitehouse MR, Lenguerrand E, Blom AW, Beswick AD (2016) Re-infection outcomes following one- and two-stage surgical revision of infected knee prosthesis: a systematic review and meta-analysis. PLoS One 11:e0151537

3. Pattyn C, De Geest T, Ackerman P, Audenaert E (2011) Preformed gentamicin spacers in two-stage revision hip arthroplasty: functional results and complications. Int Orthop 35:1471-1476
4. Parvizi J, Gehrke T, Chen AF (2013) Proceedings of the international consensus on periprosthetic joint infection. Bone Joint J 95$\mathrm{B}(11): 1450-1452$

5. Ji B, Xu B, Guo W, Rehei A, Mu W, Yang D, Cao L (2017) Retention of the well-fixed implant in the single-stage exchange for chronic infected total hip arthroplasty: an average of five years of follow-up. Int Orthop 41(5):901-909

6. Cabrita HB, Croci AT, Camargo OP, Lima AL (2007) Prospective study of the treatment of infected hip arthroplasties with or without the use of an antibiotic-loaded cement spacer. Clinics (Sao Paulo) 62:99-108

7. Jahoda D, Sosna A, Landor I, Vavrík P, Pokorný D, Hudec T (2003) Two-stage reimplantation using spacers-the method of choice in treatment of hip joint prosthesis-related infections. Comparison with methods used from 1979 to 1998. Acta Chir Orthop Traumatol Cech 70:17-24

8. Duncan CP, Beauchamp C (1993) A temporary antibiotic-loaded joint replacement system for management of complex, infections involving the hip. Orthop Clin North Am 24:751-759

9. Harris WH (1969) Traumatic arthritis of the hip after dislocation and acetabular fractures: treatment by mold arthroplasty. An endresult study using a new method of result evaluation. J Bone Joint Surg Am 51:737-755

10. Klässbo M, Larsson E, Mannevik E (2003) Hip disability and osteoarthritis outcome score. An extension of the Western Ontario and McMaster Universities Osteoarthritis Index. Scand J Rheumatol 32: 46-51

11. Hsieh PH, Shih $\mathrm{CH}$, Chang YH, Lee MS, Shih HN, Yang WE (2004) Two-stage revision hip arthroplasty for infection: comparison between the interim use of antibiotic-loaded cement beads and a spacer prosthesis. J Bone Joint Surg Am 86:1989-1997

12. Kliushin NM, Ermakov AM, Malkova TA (2017) Chronic periprosthetic hip infection: micro-organisms responsible for infection and re-infection. Int Orthop [Epub ahead of print]

13. Nelson CL, Jones RB, Wingert NC, Foltzer M, Bowen TR (2014) Sonication of antibiotic spacers predicts failure during two-stage revision for prosthetic knee and hip infections. Clin Orthop Relat Res 472:2208-2214

14. Sorlí L, Puig L, Torres-Claramunt R, González A, Alier A, Knobel H, Salvadó M, Horcajada JP (2012) The relationship between microbiology results in the second of a two-stage exchange procedure using cement spacers and the outcome after revision total joint replacement for infection: the use of sonication to aid bacteriological analysis. J Bone Joint Surg Br 94:249-253

15. Mariconda M, Ascione T, Balato G, Rotondo R, Smeraglia F, Costa GG, Conte M (2013) Sonication of antibiotic-loaded cement spacers in a two-stage revision protocol for infected joint arthroplasty. BMC Musculoskelet Disord 14:193

16. Pagnano MW, Trousdale RT, Hanssen AD (1997) Outcome after reinfection following reimplantation hip arthroplasty. Clin Orthop 338:192-204

17. Castellanos J, Flores X, Llusa M, Chiriboga C, Navarro A (1998) The Girdlestone pseudarthrosis in the treatment of infected hip replacements. Int Orthop 22:178-181

18. Schröder J, Saris D, Besselaar PP, Marti RK (1998) Comparison of the results of the Girdlestone pseudarthrosis with reimplantation of a total hip replacement. Int Orthop 22:215-218

19. Blomfeldt R, Kasina P, Ottosson C, Enocson A, Lapidus LJ (2015) Prosthetic joint infection following hip fracture and degenerative hip disorder: a cohort study of three thousand, eight hundred and seven consecutive hip arthroplasties with a minimum follow-up of five years. Int Orthop 39:2091-2096

20. Takigami I, Ito Y, Ishimaru D, Ogawa H, Mori N, Shimizu T, Terabayashi N, Shimizu K (2010) Two-stage revision surgery for hip prosthesis infection using antibiotic-loaded porous hydroxyapatite blocks. Arch Orthop Trauma Surg 130:1221-1226 